\title{
Flocking for Leader-Following Multi-Agent Systems with Time-Varying Delay
}

\author{
Fenglan Sun1, Rui Wang1, Yongfu Li², Feng Liu ${ }^{3}$ \\ ${ }^{1}$ Research Center of System Theory and Application, Chongqing University of Posts and Telecommunications, \\ Chongqing, China \\ ${ }^{2}$ College of Automation, Chongqing University of Posts and Telecommunications, Chongqing, China \\ ${ }^{3}$ School of Automation, China University of Geosciences, Wuhan, China \\ Email: sunfl@cqupt.edu.cn,w794127586@163.com
}

Received 28 November 2015; accepted 3 February 2016; published 6 February 2016

Copyright (C) 2016 by authors and Scientific Research Publishing Inc.

This work is licensed under the Creative Commons Attribution International License (CC BY).

http://creativecommons.org/licenses/by/4.0/

cC) (i) Open Access

\begin{abstract}
This paper investigates the flocking problem in multi-agent system with time-varying delay and a virtual leader. Each agent here is subject to nonlinear dynamics. For the system, the corresponding algorithm with time-varying delay is proposed. Under the assumption that the initial network is connected, it is proved that the distance between agents is in the desired distance. The theoretical deduction shows that the stable flocking motion is achieved.
\end{abstract}

Keywords

Flocking, Time-Varying Delay, Nonlinear Dynamics, Multi-Agent System, Virtual Leader

\section{Introduction}

Flocking is a collective behavior of large number of interacting agents with a common group objective. Examples of these agents include birds, fish, penguins, ants, bees, and crowds. Many scientists from rather diverse disciplines, including physics, mathematics, control engineering and biology, have been interested in flocking problem [1]-[8]. The first well-known flocking model was proposed by Craig Reynolds [2]. Reynolds started with a boid model to build a simulated flock and introduced three rules (i.e., separation, cohesion and alignment rules) for flocking. Based on Reynolds' three rules, flocking problems have been investigated from various perspectives [9]-[15]. In [16], an artificial potential function is put forward and three algorithms are introduced. It provides a theoretical framework for the designing of flocking algorithms. Multi-agent flocking under topological interactions is considered, which define a notion of hierarchical structure in the interaction graph that establish conditions building upon previous work on multi-agent systems with switching communication networks 
in [17]. Using structurally balanced signed graph theory and a specified potential function, a stable bipartite flock formation is achieved for both virtual leader and leaderless situations in [18]. However, there is a common assumption that virtual leaders guide the flocking behaviors of the group. In this paper, leader follower flocking problem of multi-agent system is considered.

A flocking problem concerning multiple leaders in which followers use the position of flocking center to keep their connections is studied in [19]. In [20], two leader-follower adaptive flocking algorithms are proposed with the combination of consensus and attraction/repulsion function respectively to solve the cohesive flocking problem and the formation flocking problem. Aiming at the group of autonomous agents consisting of multiple leader agents and multiple follower ones, a flocking behavior method with multiple leaders and a global trajectory was proposed in [21]. Yu et al., [22] give a distributed leader-follower algorithm considering the group consisting of one leader. In [23], for the circumstance with a virtual leader, the agents would follow the virtual leader and achieve the same velocity asymptotically.

In practice, time delay is inevitable and would damage the stability of system. Jing et al. [23] investigate flocking problem of multi-agent systems with time delay and discuss systems with homogeneous and inhomogeneous time delay. Yang et al. [24] proposed an adaptive flocking algorithm for multi-agent system with time delay. It is proved that the distance between agents can be larger than a constant during the motion evolution by using the flocking algorithm. The authors investigate the flocking problem of multi-agent systems led by one active virtual leader with a directed topology containing time-varying coupling delays, which based only on the three classical assumptions for flocking systems in [25]. Because of these problems, this paper will study the flocking problems in the multi-agent system with a virtual leader and time-varying delay.

The rest of this paper is organized as follows. Some basic preliminaries and flocking algorithms are presented in Section 2. Section 3 gives the nonlinear leader-following multi-agent models. Algorithms and main results are presented in Section 4. Section 5 concludes the paper and offers suggestions for future work.

\section{Preliminaries}

In this section, some related preliminary knowledge are introduced. For any vector $x$, the symbol $x^{\mathrm{T}}$ denotes its transpose and $\|x\|$ denotes the Euclidean norm. Let $\mathcal{G}=\{\mathcal{V}, \mathcal{E}, \mathcal{A}\}$ be a weighted undirected graph with the set of nodes $\mathcal{V}=\{1,2, \cdots, N\}$, and the set of agents $\mathcal{E} \subseteq \mathcal{V} \times \mathcal{V}$.

Graphs with self-loops will not be considered in this paper. The weight adjacency matrix $\mathcal{A}=\left(a_{i j}\right)_{N \times N}$, where $a_{i j} \in(0,1]$ if $(i, j) \in \mathcal{E}$, otherwise, $a_{i j}=0$. An edge denoted by the pair $(j, i)$ represents a communication link from node $j$ to $i$. A path $\mathcal{H}$ from node $i$ to node $j$ is a sequence of edges,

$\mathcal{H}=\left\{\left(i, k_{1}\right),\left(k_{1}, k_{2}\right), \cdots,\left(k_{l}, j\right)\right\} \subset \mathcal{E}$, in which all nodes $k_{l}, l=1,2, \cdots, l$ are distinct. An undirected graph is called connected if there is a path between each pair of distinct nodes. $D=\operatorname{diag}\left(d_{1}, d_{2}, \cdots, d_{N}\right)$ is the degree matrix whose diagonal elements are defined by $d_{i}=\sum_{i \neq i} a_{i j}$. The Laplacian matrix of graph $\mathcal{G}$ is $L=\left[l_{i j}\right]_{N \times N}=D-\mathcal{A} \in \mathcal{R}^{N \times N}$. Then it has following properties [26],

1) The eigenvalues of $L$ satisfy $0=\lambda_{1}(L) \leq \lambda_{2}(L) \leq \cdots \leq \lambda_{N}(L)$. If the graph $\mathcal{G}$ is connected, there is

$$
\lambda_{2}(L)=\min _{z \perp_{n}} \frac{z^{\mathrm{T}} L z}{\|z\|^{2}}>0 .
$$

2) The Laplacian matrix $L$ is a positive semi-definite matrix that satisfies the following sum-of-squares property:

$$
z^{\mathrm{T}} L z=\frac{1}{2} \sum_{i, j \in \mathcal{E}} a_{i j}\left(z_{i}-z_{j}\right)^{2}, z \in R^{n} .
$$

Lemma 1. [4] Suppose that $\mathcal{G}$ is an undirected graph of order $N$, and $\mathcal{G}_{1}$ is a graph generated by adding some edges into the graph $\mathcal{G}$. Then $\lambda_{2}\left(L\left(\mathcal{G}_{1}\right)\right) \geq \lambda_{2}(L(\mathcal{G}))$, where $L(\mathcal{G})$ and $L\left(\mathcal{G}_{1}\right)$ are the symmetric Laplacian matrices of graphs $\mathcal{G}$ and $\mathcal{G}_{1}$, respectively.

Lemma 2. For any vectors $x, y \in R^{n}$, the following matrix inequality holds: $2 x^{\mathrm{T}} y \leq x^{\mathrm{T}} x+y^{\mathrm{T}} y$.

\section{Problems Formulation}

Consider the multi-agent system described by 


$$
\left\{\begin{array}{l}
\dot{x}(t)=v_{i}(t), \\
\dot{v}_{i}(t)=f\left(v_{i}(t)\right)+u_{i}(t),
\end{array}\right.
$$

where $i=1,2, \cdots, N, x_{i} \in R^{n}, v_{i} \in R^{n}$ are the position and velocity states of $i^{\text {th }}$ agent, respectively. $f\left(v_{i}(t)\right) \in R^{n}$ is the nonlinear dynamic of agent $i$ and $u_{i} \in R^{n}$ is the control input. Denote $x_{i j}=x_{i}-x_{j},(i, j) \in \mathcal{E}$, as the relative distance between agent $i$ and agent $j$.

For the systems with virtual leader available, the dynamics of virtual leader is described as

$$
\left\{\begin{array}{l}
\dot{x}_{r}(t)=\dot{v}_{r}(t), \\
\dot{v}_{r}(t)=f\left(v_{r}(t)\right),
\end{array}\right.
$$

where $q_{r} \in R^{m}, p_{r} \in R^{m}, u_{r} \in R^{m}$ represent the position, velocity and control input vector of the virtual leader.

Assumption (A): There exists a positive constant $\rho$ satisfying

$$
\left\|f\left(z_{1}\right)-f\left(z_{2}\right)\right\| \leq \rho\left\|z_{1}-z_{2}\right\|, \forall z_{1}, z_{2} \in R^{n} .
$$

Supposed that all agents have the same sensing radius $R>0$. Then the neighboring set of agent $i$ is denoted as $N_{i}(t)=\left\{j\left\|x_{i}(t)-x_{j}(t)\right\|<R,(i, j) \in \mathcal{E}\right\}$. Since the size of agent cannot be ignored usually that a minimum allowable distance $r$ (collision distance) is considered in the model.

Definition 1: Given a constant $\varepsilon \in(0, R), \mathcal{G}(t)$ is called a dynamic undirected graph with a time-varying set of links $\mathcal{E}(t)=\{(i, j) \mid i, j \in \mathcal{V}\}$ such that

1) Initial links are generated by $\mathcal{E}(0)=\left\{(i, j) \mid r<\left\|x_{i j}(0)\right\|<R\right\}$;

2) If $(i, j) \notin \mathcal{E}(0)$ and $\left\|x_{i j}(t)\right\| \leq R-\varepsilon$, then $(i, j)$ is a new link to be added $\mathcal{E}(t)$. It is called hysteresis effect and $\gamma$ is the hysteresis distance, which is crucial in preserving connectivity of the network;

3) If $\left\|x_{i j}(t)\right\| \geq R$, then $(i, j) \notin \mathcal{E}(t)$.

The neighboring set of agent $i$ is divided into four regions, named collision region, separation region, alignment region and attraction region, in which $R>R-\varepsilon>d_{2}>d_{1}>r$. If $d_{1}<\left\|x_{i j}(t)\right\|<d_{2}$, agents $i$ and $j$ are in desired distance.

Definition 2. $\phi(\cdot)$ is a bounded function with respect to $z \in(r, R)$ between agents $i$ and $j$, which satisfies

1) $\phi(z)<0$ when $z \in\left(r, d_{1}\right)$;

2) $\phi(z)>0$ when $z \in\left(d_{2}, R\right)$;

3) $\phi(z)=0$ otherwise.

Definition 3. The pairwise bounded potential function $\psi(z):(0, \infty) \rightarrow(0, \infty)$ can be defined.

$$
\psi(z)= \begin{cases}\int_{d_{1}}^{z} \phi(s) \mathrm{d} s, & z \in\left(0, d_{1}\right), \\ 0, & z \in\left[d_{1}, d_{2}\right], \\ \int_{d_{2}}^{z} \phi(s) \mathrm{d} s, & z \in\left(d_{2}, \infty\right),\end{cases}
$$

which satisfies

1) $\psi(z)$ decreases with the increase of $z$ when $z \in\left(0, d_{1}\right)$.

2) $\psi(z)$ is increasing on $\left(d_{2}, \infty\right)$. Obviously, the potential function $\psi(z)$ reaches its minimum value 0 when $z \in\left(d_{1}, d_{2}\right)$.

According to conditions, $\phi(z)$ can be constructed as follows:

$$
\phi\left(\left\|x_{i j}\right\|\right)= \begin{cases}a_{1}\left(\left\|x_{i j}\right\|-d_{1}\right) \mathrm{e}^{\frac{\left(\left\|x_{i j}\right\|-d_{1}\right)^{2}}{b_{1}},}, & \left\|x_{i j}\right\| \in\left(r, d_{1}\right) ; \\ a_{2}\left(\left\|x_{i j}\right\|-d_{2}\right) \mathrm{e}^{\frac{\left(\left\|x_{i j}\right\| d_{2}\right)^{2}}{b_{2}}}, & \left\|x_{i j}\right\| \in\left(d_{2}, R\right) ; \\ 0, & \text { otherwise. }\end{cases}
$$

where the parameters $a_{1}, b_{1}, a_{2}, b_{2}$ are positive constants. There are $\psi(r) \geq Q_{\max }, \psi(R) \geq Q_{\max }$, and $Q_{\max }=Q_{0}+\left(N(N-1) 2-m_{0}\right) \psi(\|R-\varepsilon\|)$. 


\section{Algorithms and Main Results}

For system (1) with virtual leader (2), the flocking algorithm can be described by

$$
\begin{aligned}
u_{i}(t)= & -\sum_{j \in N_{i}(t)} \phi\left(\left\|x_{i}(t)-x_{j}(t)\right\|\right) n_{i j}(t)-c_{1} \phi\left(\left\|x_{i}(t)-x_{r}(t)\right\|\right) n_{i r}(t)-c_{2}\left(v_{i}(t)-v_{r}(t)\right) \\
& -c \sum_{j \in N_{i}(t)} a_{i j}\left(v_{i}(t)-v_{r}(t)-v_{j}(t-\tau(t))+v_{r}(t-\tau(t))\right),
\end{aligned}
$$

where $c, c_{1}, c_{2}$ are positive constants. Denote $\xi_{i}(t)=x_{i}(t)-x_{r}(t), \zeta_{i}(t)=v_{i}(t)-v_{r}(t)$, respectively. Then the system can be described by

$$
\left\{\begin{array}{l}
\dot{\xi}_{i}(t)=\zeta_{i}(t) \\
\dot{\zeta}_{i}(t)=f\left(v_{i}\right)-f\left(v_{r}\right)+u_{i}(t)
\end{array}\right.
$$

The control input (6) can be equivalently rewritten as

$$
u_{i}(t)=-\sum_{j \in N_{i}(t)} \phi\left(\left\|\xi_{i}(t)-\xi_{j}(t)\right\|\right) n_{i j}(t)-c_{1} \phi\left(\left\|\xi_{i}(t)\right\|\right) n_{i r}(t)-c_{2} \zeta_{i}(t)-c \sum_{j \in N_{i}(t)} a_{i j}\left(\zeta_{i}(t)-\zeta_{j}(t-\tau(t))\right) .
$$

Denote $\xi(t)=\left[\xi_{1}^{\mathrm{T}}, \xi_{2}^{\mathrm{T}}, \cdots, \xi_{N}^{\mathrm{T}}\right]^{\mathrm{T}}, \zeta(t)=\left[\zeta_{1}^{\mathrm{T}}, \zeta_{2}^{\mathrm{T}}, \cdots, \zeta_{N}^{\mathrm{T}}\right]^{\mathrm{T}}$.

Definition 4. Flocking motion with a virtual leader is said to be achieved asymptotically for systems (1) and (2), if for any initial state, there is $d_{1} \leq \lim _{t \rightarrow \infty}\left\|x_{i}(t)-x_{j}(t)\right\| \leq d_{2}, \quad d_{1} \leq \lim _{t \rightarrow \infty}\left\|x_{i}(t)-x_{r}(t)\right\| \leq d_{2}$, $\lim _{t \rightarrow \infty}\left\|v_{i}(t)-v_{r}(t)\right\|=0, \quad \forall i=1,2, \cdots, N$.

To demonstrate the validity of control protocol (7), the following positive semi-definite function is constructed

$$
Q=\frac{1}{2} \sum_{i=1}^{N} \sum_{j \in N_{i}} \psi\left(\left\|\xi_{i j}(t)\right\|\right)+\frac{1}{2} \sum_{i=1}^{N} \zeta_{i}^{\mathrm{T}}(t) \zeta_{i}(t)+\frac{1}{2} \sum_{i=1}^{N} c_{1} \int_{d_{2}}^{\xi_{i}} \phi(s) \mathrm{d} s+\sum_{i=1}^{N} \int_{t-\tau(t)}^{t} \zeta_{i}^{\mathrm{T}}(s) \zeta_{i}(s) \mathrm{d} s,
$$

where $\xi_{i j}(t)=\xi_{i}(t)-\xi_{j}(t)$.

Theorem 1. Consider a multi-agent system modeled by dynamics (1) and (2), driven by control protocol (5). Suppose that the network is initially connected and $Q_{0}:=Q(\xi(0), \zeta(0))$ is bounded. When

$\dot{\tau}(t) \leq 1-\frac{2 \rho-c_{2}-c \lambda_{2}+1+\frac{1}{2} c k \lambda_{N}+\frac{1}{2} c \lambda_{N}}{k}$, then following statements hold,

1) $G(t)$ is connected for all $t \geq 0$;

2) No collision occurs among agents for all $t \geq 0$;

3) Flocking motion with a virtual leader is achieved asymptotically.

Proof: Denote the topology switching time sequence as $t_{k}, k \in \mathcal{Z}$. Without loss of generality, assume $t_{0}=0$. Taking the time derivative of the Lyapunov function $Q$ on $t \in\left[t_{0}, t_{1}\right)$ gives

$$
\begin{aligned}
\dot{Q}= & \sum_{i=1}^{N} \zeta_{i}^{\mathrm{T}}(t) \sum_{j \in N_{i}(t)} \phi\left(\left\|\xi_{i j}(t)\right\|\right) n_{i j}(t)+c_{1} \sum_{i=1}^{N} \zeta_{i}^{\mathrm{T}}(t) \phi\left(\left\|\xi_{i}(t)\right\|\right) n_{i r}(t)+\sum_{i=1}^{N} \zeta_{i}^{\mathrm{T}}(t) \dot{\zeta}_{i}(t) \\
& +\sum_{i=1}^{N}\left(\zeta_{i}^{\mathrm{T}}(t) \zeta_{i}(t)-(1-\dot{\tau}(t)) \zeta_{i}^{\mathrm{T}}(t-\tau(t)) \zeta_{i}(t-\tau(t))\right) \\
= & -\sum_{i=1}^{N}\left(\zeta_{i}^{\mathrm{T}}(t) \zeta_{i}(t)-(1-\dot{\tau}(t)) \zeta_{i}^{\mathrm{T}}(t-\tau(t)) \zeta_{i}(t-\tau(t))\right) \\
& +\sum_{i=1}^{N} \zeta_{i}^{\mathrm{T}}(t)\left(f\left(v_{i}\right)-f\left(v_{r}\right)-\sum_{j \in N_{i}(t)} \phi\left(\left\|\xi_{i}(t)-\xi_{j}(t)\right\|\right) n_{i j}(t)-c_{1} \phi\left(\left\|\xi_{i}(t)\right\|\right) n_{i r}(t)\right. \\
& \left.-c_{2} \zeta_{i}(t)-c \sum_{j \in N_{i}(t)} a_{i j}\left(\zeta_{i}(t)-\zeta_{j}(t-\tau(t))\right)\right) \\
& +\sum_{i=1}^{N}\left(\zeta_{i}^{\mathrm{T}}(t) \zeta_{i}(t)-(1-\dot{\tau}(t)) \zeta_{i}^{\mathrm{T}}(t-\tau(t)) \zeta_{i}(t-\tau(t))\right) .
\end{aligned}
$$


From Lemma 2, there is

$$
\begin{aligned}
\dot{Q} \leq & 2 \rho\|\zeta(t)\|^{2}-c_{2}\|\zeta(t)\|^{2}-c \lambda_{2}(L)\|\zeta(t)\|^{2}+\frac{1}{2} c \lambda_{N}(L)\|\zeta(t)\|^{2} \\
& +\frac{1}{2} c \lambda_{N}(L)\|\zeta(t-\tau(t))\|^{2}+\|\zeta\|^{2}-(1-\dot{\tau}(t))\|\zeta(t-\tau(t))\|^{2} .
\end{aligned}
$$

For a positive constant $k$, one has $\|\zeta(t-\tau(t))\|^{2} \leq k\|\zeta(t)\|^{2}$, thus

$$
\begin{aligned}
& \qquad \begin{array}{r}
\dot{Q} \leq 2 \rho\|\zeta(t)\|^{2}-c_{2}\|\zeta(t)\|^{2}-c \lambda_{2}(L)\|\zeta(t)\|^{2}+\frac{1}{2} c \lambda_{N}(L)\|\zeta(t)\|^{2} \\
+\frac{1}{2} c k \lambda_{N}(L)\|\zeta(t)\|^{2}+\|\zeta(t)\|^{2}-(1-\dot{\tau}(t))\|\zeta(t-\tau(t))\|^{2}
\end{array} \\
& \text { Assume that } \tau(t) \text { satisfy } \dot{\tau}(t) \leq 1-\frac{2 \rho-c_{2}-c \lambda_{2}+1+\frac{1}{2} c k \lambda_{N}+\frac{1}{2} c \lambda_{N}}{k} \text {, there is } \\
& \dot{Q} \leq 0, \forall t \in\left[t_{0}, t_{1}\right),
\end{aligned}
$$

which implies that

$$
Q(t) \leq Q_{0}<\infty, \forall t \in\left[t_{0}, t_{1}\right) .
$$

By definition (2), one has $\psi(\|R\|) \geq Q_{\max }$. Therefore, no edge distance will be tend to $R$ for $\forall t \in\left[t_{0}, t_{1}\right)$, implying that no existing edges will be lost before time $t_{1}$. Hence, new edges must be added into the network at $t_{1}$. For a system consists of $N$ agents, there are at most $N(N-1) / 2$ edges. At the initial instant $t_{0}$, the system consists of $m_{0}$ edges, then

$$
Q\left(t_{k}\right) \leq Q_{0}+\left(N(N-1) / 2-m_{0}\right) \psi(\|R-\varepsilon\|)=Q_{\max } \cdot
$$

Hence there is no edge lost. In addition, from the definite of potential function, one has $\psi(\|r\|) \geq Q_{\max }$. Therefore, no collision occurs during $\left[t_{0}, t_{1}\right)$.

Similar to the above analysis, taking the time derivative of $Q(t)$ on every $\left[t_{k-1}, t_{k}\right)$. By lemma 1 , there is

$$
\dot{\tau}(t) \leq 1-\frac{2 \rho-c_{2}-c \lambda_{2}(L(0))+1+c \lambda_{N}(L(0)) k}{k} \leq 1-\frac{2 \rho-c_{2}-c \lambda_{2}(L(t))+1+c \lambda_{N}(L(t)) k}{k}
$$

one has $\dot{Q}(t) \leq 0$, which implies $Q(t) \leq Q\left(t_{k-1}\right)<\infty, \forall t \in\left[t_{k-1}, t_{k}\right)$. Thus no edge distance will tend to $R$ for $\left[t_{k-1}, t_{k}\right)$, implying that no edge will be lost before time $t_{k}$ and $Q\left(t_{k}\right)$ is finite. Since $\mathcal{G}(0)$ is connected and no edge in $E(0)$ is lost, $\mathcal{G}(t)$ will be connected for all $t \geq 0$. This completes the proof of part (1).

Similarly, from $\psi(\|r\|) \geq Q_{\max }$, deducing that no edge distance will tend to $r$, for all $t \geq 0$. Thus collision is avoided during the whole process. This completes the proof of part (2). To proof part (3), assume that there are $m_{k}$ new edges being added to the evolving network at time $t_{k}$. As no edges are lost for $t \geq 0$, and $m_{k} \leq N(N-1) / 2-m_{0} \leq(N-1)(N-2) / 2$. Therefore, the number of switching times of the system (1) is finite, which implies that the evolving network $\mathcal{G}(t)$ eventually becomes fixed. Denote the last topology switching as $t_{k}$. Then $Q$ is continuous and monotonously decreasing for $t \in\left[t_{k}, \infty\right)$. Hence the set

$$
\Omega:=\left\{\xi \in D, \zeta \in R^{N n} \mid Q(\xi, \zeta) \leq Q^{*}\right\},
$$

is positively invariant, where

$$
D=\left\{\xi \in R^{N^{2} n}\left\|\xi_{i j}\right\| \in\left[\min \left(\psi^{-1}\left(Q_{\max }\right)\right), \max \left(\psi^{-1}\left(Q_{\max }\right)\right)\right],(i, j) \in \mathcal{E}(t), t \in\left[t_{k}, \infty\right)\right\},
$$

and $\xi=\left(\xi_{11}, \cdots, \xi_{1 N}, \cdots, \xi_{N 1}, \cdots, \xi_{N N}\right)^{\mathrm{T}} \in R^{N n}$.

Since $\mathcal{G}(t)$ is connected for all $t \geq 0$, one has $\left\|\xi_{i j}\right\| \leq(N-1) R$, for all $(i, j) \in \mathcal{E}(t)$. As $Q(t) \leq Q_{\max }$, one has $\zeta_{i}^{\mathrm{T}} \zeta_{i} \leq 2 Q_{\max }$. thus $\left\|\zeta_{i}(t)\right\| \leq \sqrt{2 Q_{\max }}$. Therefore, the set $\Omega$ is compact. It follows from LaSalle's invariance principle that if the initial condition lies in $\Omega$, then the corresponding trajectories will converge to the largest in- 
variant set inside the region

$$
S=\left\{\xi \in R^{N^{2} n}, \zeta \in R^{N n} \mid \dot{Q}(\xi, \zeta)=0\right\} .
$$

From (8), $\dot{Q}=0$ if and only if $v_{1}=\cdots=v_{N}=v_{r}$, which implies that the velocities of all agents will converge to that of the virtual leader asymptotically.

Since $v_{1}=\cdots=v_{N}=v_{r}$, there is $\dot{\zeta}_{i}=0$ for all $i=1,2, \cdots, N$. From (6), one has

$$
\begin{aligned}
\dot{\zeta}_{i}(t) & =f\left(v_{i}\right)-f\left(v_{r}\right)+u_{i}(t) \\
& =-\sum_{j \in N_{i}(t)} \phi\left(\left\|x_{i}(t)-x_{j}(t)\right\|\right) n_{i j}(t)-c_{1} \phi\left(\left\|x_{i}(t)-x_{r}(t)\right\|\right) n_{i r}(t) \\
& =0 .
\end{aligned}
$$

Thus, unless the inital configuration of the agents is close enough to the global minimum, almost every final configuration locally minimizes each agent's global potential. which implies

$$
\begin{aligned}
& \left\|x_{i}-x_{j}\right\| \in\left[d_{1}, d_{2}\right] ; \\
& \left\|x_{i}-x_{r}\right\| \in\left[d_{1}, d_{2}\right] .
\end{aligned}
$$

Then the flocking is achieved. This completes the proof of part (3), thus Theorem 1 hold.

Remark 1. If $\tau(t)=\tau$ is the constant delay, from the deduction above, Theorem 1 is also hold.

\section{Conclusion}

This paper mainly discusses the flocking problem of multi-agent system with a virtual leader and time-varying delay. Unlike most existing flocking algorithms, each agent here is subject to nonlinear dynamics. The corresponding algorithms with the time-varying delay are proposed. Under the assumption that the initial network is connected, the theoretical deduction is made. The related topic over the directed network or the jointly connected network will be studied in future.

\section{Acknowledgements}

We thank the Editor and the referee for their comments. This work was supported by the National Nature Science Foundation of China under Grants 61503053, 61472374 and 61304197, the Natural Science Foundation Project of CQ CSTC, China (Grant No. cstc2013jcyjA40018), the Youth Science Research Project of CQUPT, China (Grant Nos. A2012-78 and A2012-82), the Doctor Start-up Foundation of CQUPT, China (Grant Nos. A2012-23 and A2012-26), the Natural Science Fundation of CQJW, China (Grant Nos. KJ130506 and KJ1400435), and Training Programme Foundation for the Talents of Higher Education Commission. This support is greatly appreciated.

\section{References}

[1] Toner, J. and Tu, Y. (1998) Flocks, Herds, and Schools: A Quantitative Theory of Flocking. Physical Review E, 58, 4828. http://dx.doi.org/10.1103/PhysRevE.58.4828

[2] Reynolds, C.W. (1987) Flocks, Herds and Schools: A Distributed Behavioral Model. ACM Siggraph Computer Graphics, 21, 25-34. http://dx.doi.org/10.1145/37402.37406

[3] Toner, J. and Tu, Y. (2005) Hydrodynamics and Phases of Flocks. Annals of Physics, 318, 170-244. http://dx.doi.org/10.1016/j.aop.2005.04.011

[4] Olfati-Saber, R. (2006) Flocking for Multi-Agents with a Virtual Leader. IEEE Transactions on Automatic Control, 51, 410-420.

[5] Crowther, B. (2002) Flocking of Autonomous Unmanned Air Vehicles. Aeronautical Journal, 107, 99-109.

[6] Akyildiz, I.F., Su, W. and Cayirci, E. (2002) A Survey on Sensor Networks. IEEE Communications Magazine, 40, 102-114. http://dx.doi.org/10.1109/MCOM.2002.1024422

[7] Partridge, B.L. (1984) The Chorus-Line Hypothesis of Maneuver in Avian Flocks. Nature, 309, 344-345.

[8] Gazi, V. and Passino, K.M. (2004) Stability Analysis of Social Foraging Swarms. IEEE Transactions on Systems, Man, and Cybernetics, Part B: Cybernetics, 34, 539-557. http://dx.doi.org/10.1109/TSMCB.2003.817077 
[9] Ren, W. and Atkins, E. (2007) Distributed Multi-Vehicle Coordinated Control via Local Information Exchange. International Journal of Robust and Nonlinear Control, 17, 1002-1033. http://dx.doi.org/10.1002/rnc.1147

[10] Tanner, H.G., Jadbabaie, A. and Pappas, G.J. (2007) Flocking in Fixed and Switching Networks. IEEE Transactions on Automatic Control, 52, 863-868. http://dx.doi.org/10.1109/TAC.2007.895948

[11] Su, H., Wang, X. and Lin, Z. (2009) Flocking of Multi-Agents with a Virtual Leader. IEEE Transactions on Automatic Control, 54, 293-307. http://dx.doi.org/10.1109/TAC.2008.2010897

[12] Topaz, C.M. and Bertozzi, A.L. (2004) Swarming Patterns in a Two-Dimensional Kinematic Model for Biological Groups. SIAM Journal on Applied Mathematics, 65, 152-174. http://dx.doi.org/10.1137/S0036139903437424

[13] Chen, Z. and Zhang, H.T. (2013) A Remark on Collective Circular Motion of Heterogeneous Multi-Agents. Automatica, 49, 1236-1241. http://dx.doi.org/10.1016/j.automatica.2013.01.017

[14] Zhang, H.T., Zhai, C. and Chen, Z. (2011) A General Alignment Repulsion Algorithm for Flocking of Multi-Agent Systems. IEEE Transactions on Automatic Control, 56, 430-445. http://dx.doi.org/10.1109/TAC.2010.2089652

[15] Lu, X.Q., Austin, F. and Chen, S.H. (2011) Flocking in Multi-Agent Systems with Active Virtual Leader and TimeVarying Delays Coupling. Communications in Nonlinear Science and Numerical Simulation, 16, 1014-1026. http://dx.doi.org/10.1016/j.cnsns.2010.05.004

[16] Olfati-Saber, R. (2006) Flocking for Multi-Agent Dynamic Systems: Algorithms and Theory. IEEE Transactions on Automatic Control, 51, 401-420. http://dx.doi.org/10.1109/TAC.2005.864190

[17] Martin, S. (2014) Multi-Agent Flocking under Topological Interactions. Systems and Control Letters, 69, 53-61. http://dx.doi.org/10.1016/j.sysconle.2014.04.004

[18] Fan, M.C., Zhang, H.T. and Wang, M. (2014) Bipartite Flocking for Multi-Agent Systems. Communications in Nonlinear Science and Numerical Simulation, 19, 3313-3322. http://dx.doi.org/10.1016/j.cnsns.2013.10.009

[19] Gu, D. and Wang, Z. (2009) Leader-Follower Flocking: Algorithms and Experiments. IEEE Transactions on Control Systems Technology, 17, 1211-1219.

[20] Jia, Y. and Zhang, W. (2014) Distributed Adaptive Flocking of Robotic Fish System with a Leader of Bounded Unknown Input. International Journal of Control, Automation and Systems, 12, 1049-1058. http://dx.doi.org/10.1007/s12555-013-0518-6

[21] Li, M., Liang, J. and Li, S. (2014) Flocking Behavior with Multiple Leaders and Global Trajectory. Journal of Central South University, 21, 2324-2333. http://dx.doi.org/10.1007/s11771-014-2184-0

[22] Yu, W., Chen, G. and Cao, M. (2010) Distributed Leader-Follower Flocking Control for Multi-Agent Dynamical Systems with Time-Varying Velocities. Systems \& Control Letters, 59, 543-552. http://dx.doi.org/10.1016/j.sysconle.2010.06.014

[23] Luo, J., Zhang, S., Kang, H., et al. (2013) Flocking Algorithms for Multi-Agent Systems with Time-Delay. Proceedings of the IEEE International Workshop on Microwave and Millimeter Wave Circuits and System Technology (MMWCST), Chengdu, 24-25 October 2013, 428-431.

[24] Yang, Z., Zhang, Q. and Chen, Z. (2013) A Novel Adaptive Flocking Algorithm for Multi-Agents System with Time Delay and Nonlinear Dynamics. Proceedings of the 32nd IEEE Chinese Control Conference (CCC), Xi'an, 26-28 July 2013, 998-1001.

[25] Lu, X., Austin, F. and Chen, S. (2011) Flocking in Multi-Agent Systems with Active Virtual Leader and Time-Varying Delays Coupling. Communications in Nonlinear Science and Numerical Simulation, 16, 1014-1026. http://dx.doi.org/10.1016/j.cnsns.2010.05.004

[26] Shi, H., Wang, L. and Chu, T. (2006) Virtual Leader Approach to Coordinated Control of Multiple Mobile Agents with Asymmetric Interactions. Physica D: Nonlinear Phenomena, 213, 51-65.

http://dx.doi.org/10.1016/j.physd.2005.10.012 